\title{
Parallel Projection of Amplitude and Phase Information from the Hindbrain to the Midbrain of the African Electric Fish Gymnarchus niloticus
}

\author{
Masashi Kawasaki and Yuan-Xing Guo \\ Department of Biology, University of Virginia, Charlottesville, Virginia 22903
}

Two distinct sensory cues in electrosensory signals, amplitude modulation and differential phase modulation, are essential for an African wave-type electric fish, Gymnarchus, to perform the jamming avoidance responses. Individual neurons in the first brain station for central processing, the electrosensory lateral line lobe (ELL), were investigated by the in vivo whole-cell recording and labeling technique for their physiological responses, location, morphology, and projection areas.

Neurons in the dorsal zone of the ELL responded selectively to amplitude modulation. Neurons in the outer cell layer of the medial zone were categorized physiologically into two groups: amplitude-sensitive and differential phase-sensitive. All but one neuron in the inner cell layer of the medial zone responded exclusively to differential phase modulation. All neurons recorded and labeled in the ELL had pyramidal morphology with large and extensive apical dendrites and less extensive basal dendrites. They were found to project to two midbrain nuclei: the nucleus praeeminentialis and the torus semicircularis. Amplitude-sensitive neurons in the dorsal zone projected exclusively to the lateral posterior subdivision, the torus semicircularis. Neurons in the medial zone projected to the medial dorsal and lateral anterior subdivisions of the torus semicircularis.

Although some neurons in the ELL responded to both amplitude and differential phase modulation, they did not differentiate between temporal patterns of the two cues that encode necessary information for the jamming avoidance response. Overlapping projection of amplitude and differential phase-sensitive neurons to the torus semicircularis suggests integration of the two sensory cues in this nucleus.

Key words: electric fish; jamming avoidance response; phase comparison; binaural comparison; whole-cell recording; parallel projection
The African weakly electric fish Gymnarchus niloticus emits wavetype electric organ discharges (EODs) from the electric organ in the tail for electrolocation. The EODs are sensed by electroreceptors located all over the body surface for electrolocation (Lissmann, 1958; Lissmann and Machin, 1958) and electrocommunication (Hopkins, 1974). The frequency of EOD is normally stable and fluctuates only a few Hertz around an individually fixed frequency that ranges from 300 to $500 \mathrm{~Hz}$ (Bullock et al., 1975). When two individuals with close discharge frequencies meet, however, they shift their discharge frequencies away from each other to create a larger frequency difference (Bullock et al., 1975). This behavior, the jamming avoidance response (JAR), has a function of avoiding mutual interference effects in their electrolocation (Heiligenberg, 1975). When they shift their discharge frequencies, a decision is made as to which way to change the discharge frequency, upward or downward, without a trial-anderror behavior. This means that fish are able to determine the sign of frequency difference, i.e., whether the frequency of a neighbor is higher or lower than their own, within the latency of this behavior, which is approximately a few hundred milliseconds.

Received May 26, 1998; revised July 6, 1998; accepted July 9, 1998.

This study was supported by National Institute of Mental Health Grant R29 MH48115-01A1, Research Scientist Development Award K-02 MH01256-01 from the Alcohol, Drug Abuse, and Mental Health Administration, and National Science Foundation Grant IBN9631785 to M.K. We thank Yasuko Kawasaki for preparation of figures. We thank two anonymous referees for critical comments and Cameron McLaughlin for editing English.

Correspondence should be addressed to Masashi Kawasaki, Department of Biology, Gilmer Hall, University of Virginia, Charlottesville, VA 22903.

Copyright (C) 1998 Society for Neuroscience $\quad 0270-6474 / 98 / 187599-13 \$ 05.00 / 0$
In the JAR, the fish does not use the information about its own discharge frequency, which is available at the pacemaker nucleus in the brain that drives the EOD of that fish (Kawasaki, 1994). Instead, the fish analyzes a complex signal pattern that is created by an addition of electrosensory feedback from its own discharge and the EOD of a neighbor to examine the frequency relation between them. Kawasaki (1993) identified a computational algorithm with which the fish derives the sign of the frequency difference between two fish from the signal mixture. The signal mixture exhibits periodic changes in two parameters: amplitude and phase difference. Both amplitude and phase difference modulate at a frequency equal to the absolute value of the frequency difference. Thus, either amplitude or phase difference alone cannot unambiguously represent the sign of frequency differences. The temporal pattern of amplitude and differential phase modulations taken together uniquely represents the sign of the frequency difference because the timing of the phase modulation changes relative to the amplitude modulation contingent on the sign of the frequency difference. This algorithm suggests that amplitude and phase information are separately processed and subsequently integrated.

Two classes of electroreceptors, O-type and S-type, sample amplitude and phase information, respectively, in Gymnarchus (Bullock et al., 1975). Primary afferent fibers from these electroreceptors terminate in the first brain station, the electrosensory lateral line lobe (ELL) in the medulla. Using the in vivo wholecell recording and labeling method (Rose and Fortune, 1996), physiological and morphological characteristics of neurons in the ELL were studied in the present report, and the sensitivities of 
neurons to complex patterns of amplitude modulation and phase modulation were examined. The dorsal zone (DZ) of the ELL was found to contain neurons that are sensitive only to amplitude modulation, whereas the medial zone (MZ), in which phasesensitive neurons were previously found (Kawasaki and Guo, 1996), was found to contain also neurons sensitive only to amplitude modulation. Thus, amplitude and phase information are processed separately, and their nonlinear integration, which is required for JAR, does not appear to occur in the ELL. Projection areas of these neurons, however, showed significant overlap in midbrain nuclei, suggesting that amplitude and phase information are integrated there.

\section{MATERIALS AND METHODS}

Animals. Approximately 80 Gymnarchus niloticus (11-17 cm) were used. All experiments were approved by the University of Virginia Animal Care Committee. Environmental conditions in the holding tanks were identical to those described in Kawasaki (1994). Intramuscular injection of Flaxedil (gallamine triethiodide, $0.1 \%, 3-6 \mu \mathrm{l}$ ) after initial and temporal anesthesia with MS-222 $(1: 10,000)$ immobilized the fish and greatly attenuated amplitude of electric organ discharges. Oxygen-saturated water was provided to the gill with a tube inserted in the mouth. Activity of the pacemaker command signal was recorded to monitor the condition of the fish throughout experiments.

After local application of Xylocaine (2\%), a small hole was drilled in the skull above the corpus cerebelli. The ELL was exposed by removing the caudal edge of the corpus cerebelli with fine suction tubing. Fish were gently held with a sponge-lined clamp and submerged in water except for a small area around the skull opening.

Whole-cell recording. Whole-cell recording and labeling was performed according to Rose and Fortune (1996). A glass capillary of $1.2 \mathrm{~mm}$ outer diameter (World Precision Instruments, catalog \#1B120F) was pulled with three steps on a Flaming-Brown type micropipette puller (Sutter, model P-97). A tip outer diameter of $\sim 1.2 \mu \mathrm{m}$ yielded best results. These electrodes showed $\sim 20 \mathrm{M} \Omega$ of resistance when filled with biocytincontaining intracellular solution as described in Rose and Fortune (1996).

Positive pressure was applied to the electrode while advancing it into brain tissue to prevent the tip from clogging. After reaching a recording area, pressure was released, and the electrode was slowly advanced (Burleigh microdrive) with a search stimulus (see below). Meanwhile, square current pulses $(2 \mathrm{~Hz},+0.1 \mathrm{nA}$, offset $+0.05 \mathrm{nA})$ were passed through the electrode to monitor the electrode and seal resistance. When seal resistance increased to $\sim 300 \mathrm{M} \Omega$, gentle negative pressure was applied to the electrode. When electrode geometry matched the underlying membrane, a high-resistance seal (1-2 G $\Omega$ ) was easily established. The pressure was immediately released, and steady negative current of 1-2 nA was then applied to perforate the patch membrane. Resting potential was typically $40-50 \mathrm{mV}$, and spike height was typically $30 \mathrm{mV}$ but often exceeded $60 \mathrm{mV}$. Postsynaptic potentials of $20 \mathrm{mV}$ were commonly seen. Neurons could be held for hours but were typically studied for 30 minutes. Clear action potentials could be recorded extracellularly before making the high-resistance seal, allowing us to access physiological response properties of a neuron before making the decision to attempt to establish a high-resistance seal.

After physiological recordings, sinusoidal current $(1 \mathrm{~Hz},+2 \mathrm{nA}$, offset, $+1 \mathrm{nA}$ ) was passed to inject biocytin into the cell for several minutes. In most cases, only one neuron was injected with biocytin for anatomical labeling in either side of the brain for unambiguous matching of physiological and morphological data. A great majority ( $>90 \%)$ of physiologically recorded neurons were unambiguously labeled by biocytin. Survival time of $8 \mathrm{hr}$ was necessary to completely fill long projecting axons (up to $7 \mathrm{~mm}$ ). Fish were deeply anesthetized with MS-222 (1:1000) and perfused with $4 \%$ paraformaldehyde. Histological sections were processed with a standard ABC-DAB method as in Kawasaki (1994).

Sensory stimulus. Responsiveness of neurons to amplitude modulation and differential phase modulation was first tested with the $S_{1} / S_{2}$ stimulus regimen in which signals mimicking the EODs of the animal $\left(S_{1}\right)$ and of a neighbor $\left(S_{2}\right)$ were presented through two pairs of electrodes (Kawasaki, 1993). A sinusoidal signal, $S_{1}$, was applied between an electrode inserted in the mouth and an electrode placed near the tail. A sinusoidal signal, $\mathrm{S}_{2}$, was applied between two electrodes straddling the prepara- tion. In this $S_{1} / S_{2}$ regimen, sinusoidal modulations of amplitude and differential phase result because of the addition of $S_{1}$ and $S_{2}$ in the tank. These amplitude and differential phase modulations were, however, interlocked so that differential phase modulation alone could not be created. Nevertheless, effects of amplitude or differential phase modulation could be derived by comparing two histograms with different signs of frequency differences (Df) between $\mathrm{S}_{1}$ and $\mathrm{S}_{2}\left(\mathrm{Df}=f_{2}-f_{1} ; f_{1}\right.$, frequency of $\mathrm{S}_{1} ; f_{2}$, frequency of $\mathrm{S}_{2}$ ), because amplitude and differential phase modulation show unique temporal patterns for different signs of Df (Rose and Heiligenberg, 1985; Kawasaki, 1993). Stimulus amplitude of $S_{1}$ was set at $1-2 \mathrm{mV} / \mathrm{cm}$ measured at the gill cover. $S_{2}$ amplitude was set to $\sim 30 \%$ of $S_{1}$ measured at the same location. By the $S_{1} / S_{2}$ regimen, we found that some neurons in the ELL responded to both amplitude and differential phase modulation. These neurons were further examined using the phase chamber in which the head and trunk of the fish were electrically isolated (Heiligenberg and Rose, 1985; Kawasaki, 1993). With this phase-chamber regimen, amplitude and phase could be independently controlled. Stimulus amplitude was set at $1-2 \mathrm{mV} / \mathrm{cm}$ at the gill and $3-5 \mathrm{mV} / \mathrm{cm}$ at the trunk. For both types of stimulus regimen, sinusoidal waves with and without amplitude and phase modulations were digitally created by a computer (Gateway, model P5-133) equipped with a digital-to-analog converter (Tucker Davis Technology, model DA3-4). Stimuli were delivered to the experimental tanks via homemade isolators with field effect transistors. Frequency of the signal mimicking the fish's own signal was set within $20 \mathrm{~Hz}$ of the EOD frequency of the fish measured before curarization. Water resistivity was $\sim 5 \mathrm{k} \Omega \cdot \mathrm{cm}$ for all experiments.

Analysis of responses. Spike histograms were constructed against one period of stimulus modulation (typically $1-4 \mathrm{~Hz}$ ). For the $S_{1} / S_{2}$ regimen, histograms for $-\mathrm{Df}$ and $+\mathrm{Df}$ had identical and synchronous sinusoidal modulation for amplitude, but $180^{\circ}$-shifted functions of differential phase modulation (Rose and Heiligenberg, 1985) (see Fig. 2). A scalar value, $p$, was computed as:

$$
p=\arctan \left(\sum_{i=1}^{n} x_{i} \cdot \sin _{i} / n / \sum_{i=1}^{n} x_{i} \cdot \cos _{i} / n\right)
$$

to assess the position of the center of responses in a histogram. $p=0^{\circ}$, and $360^{\circ}$ corresponds to the beginning and the end of the modulation cycle histogram period, and $180^{\circ}$ corresponds to the middle of histograms.

The degree of synchronization of spike occurrence to the cycle of carrier signal, vector strength (v) (Goldberg and Brown, 1969), was computed as:

$$
v=\sqrt{\left(\sum_{i=1}^{n} x_{i} \cdot \cos _{i} / n\right)^{2}+\left(\sum_{i=1}^{n} x_{i} \cdot \sin _{i} / n\right)^{2}}
$$

$v=0$ and $v=1$, respectively, corresponding to no and complete synchronization.

In the two equations above, $x_{i}$ is the spike count of the $i$ th bin in histograms. $\cos _{i}=\cos (i \cdot 2 \pi / n), \sin _{i}=\sin (i \cdot 2 \pi / n), i=1, \ldots, n . n$ is the total number of bins, which is 20 or 50 for Equation 1 , and is $1 / f \times 10^{6}$ ( $f$, frequency of the carrier signal) for Equation 2.

\section{RESULTS}

\section{Overview of the hindbrain and midbrain of Gymnarchus}

Figure 1 shows major structures of the electrosensory system in the hindbrain and midbrain that are involved in this study. Whole-cell recording and labeling was performed in the ELL, which consists of large bilateral lobes in the hindbrain. Each lobe contains three zones: medial (MZ), dorsal (DZ), and ventral (VZ), each of which is layered. The deep fiber layer (DFL), the deepest layer, constitutes the core of the ELL and is shared by all zones. In the MZ, DFL is covered with an inner cell layer (ICL), outer fiber layer (OFL), outer cell layer (OCL), and molecular layer (ML), in that order from the core. In DZ and VZ, DFL is covered only with OCL and ML. OCL and ML are continuous 

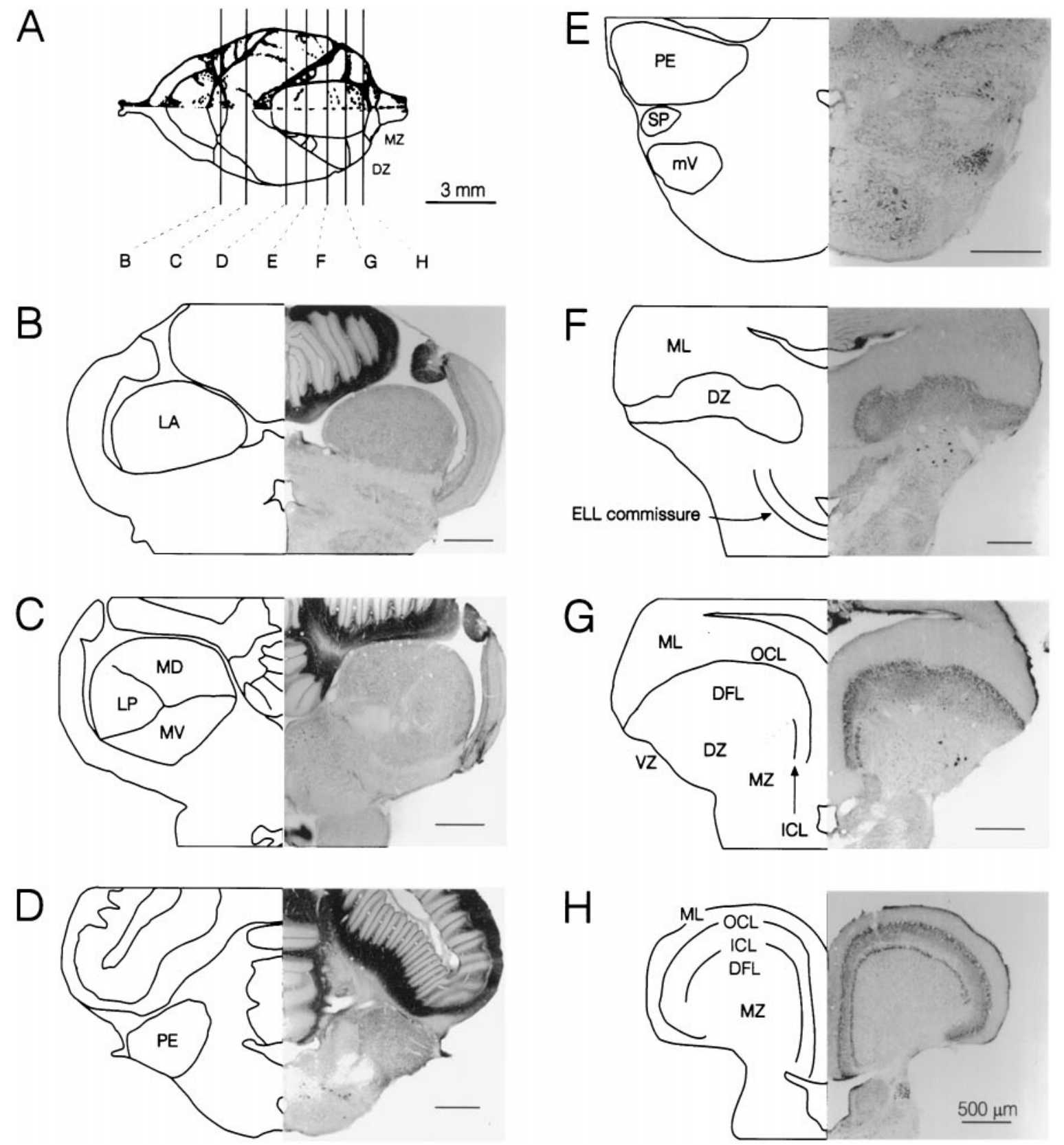

Figure 1. Overview of the hindbrain and midbrain of Gymnarchus. $A$, Dorsal view showing locations of transverse sections $(B-H)$. $B, C$, Torus semicircularis is subdivided into four divisions: $L A, M D, L P$, and $M V . D, E$, PE lies caudoventral to the torus. $F-H$, ELL. DZ of ELL starts at level of $F$, and MZ starts at level of $G$. In $H$, MZ occupies most of ELL. $S P$, Nucleus subpraeeminentialis.

throughout all three zones. Intracellular fills with biocytin revealed projection areas in two midbrain structures: the nucleus praeeminentialis (PE) and the torus semicircularis (hereafter torus). The torus consists of four distinct subdivisions: lateral anterior (LA), lateral posterior (LP), medial dorsal (MD), and medial ventral (MV). Anatomical nomenclature of brain structures of Gymnarchus in this paper follows that of Bass and Hopkins (1982).

High-resistance seal was readily established on neurons in the two layers, OCL and ICL, of the DZ and MZ. No attempt was made to establish high-resistance seal in the ML and the DFL to avoid clogging of electrodes. We recorded and labeled 24 neurons in the DZ and 71 neurons in the MZ using the $S_{1} / S_{2}$ stimulus regimen and six neurons in the $\mathrm{DZ}$ and 25 neurons in the $\mathrm{MZ}$ using the phase chamber.

\section{Experiments with $S_{1} / S_{2}$ stimuli}

Neurons in DZ

All recorded and labeled neurons in DZ $(n=24)$ were identified in OCL. Seventeen of 24 were sensitive to amplitude modulation of the carrier signal (tuberous type), and seven were sensitive to low frequency $(<50 \mathrm{~Hz})$ carrier signal (ampullary type, see a designated section for ampullary neurons below).

The tuberous-type neurons responded to amplitude modulation of $S_{1}$. When $S_{2}$ was added to create differential phase modulation in addition to amplitude modulation, neurons still re- 

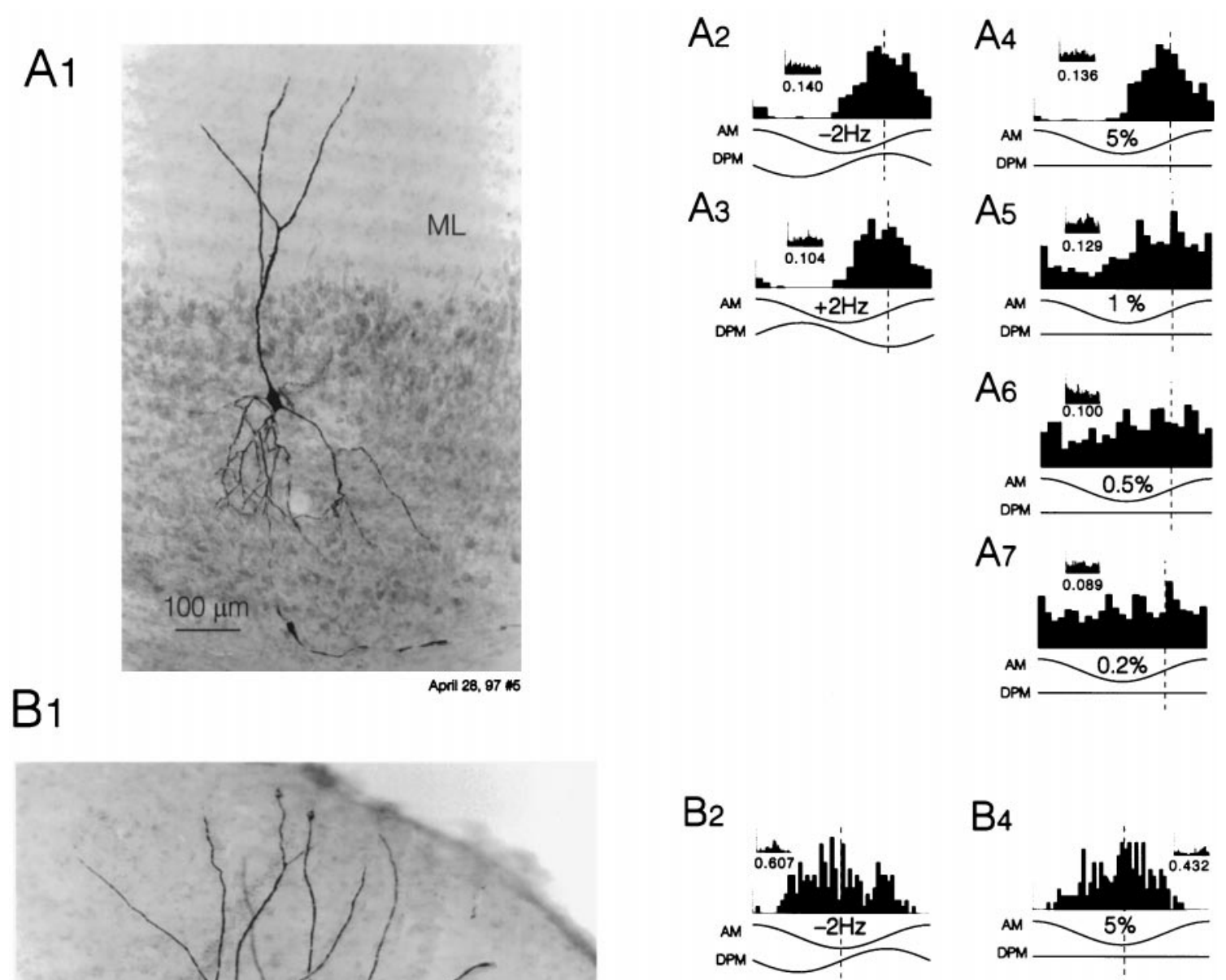

$\mathrm{B} 4$
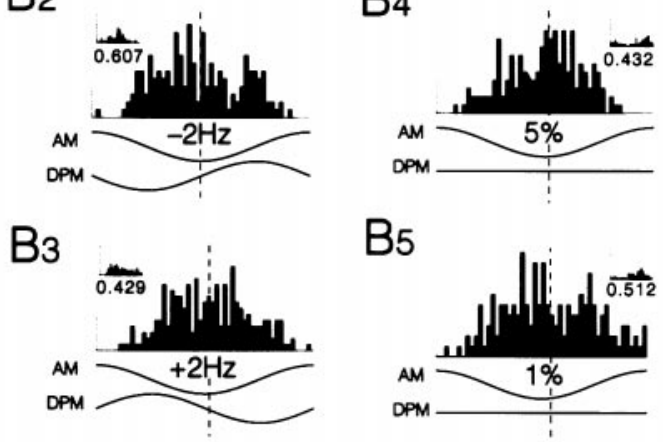

B5
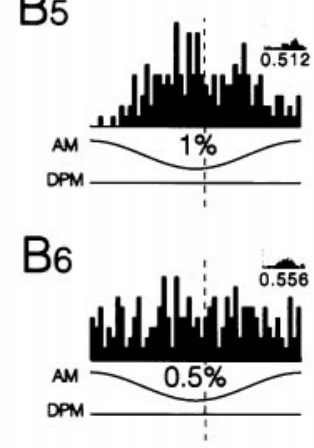

Figure 2. All neurons found in DZ were sensitive only to amplitude modulation. Montage photomicrograph is shown at left, response histograms of corresponding neurons at right. Histograms were constructed against modulation cycles $(\sim 30 \mathrm{cycles}, 2 \mathrm{~Hz})$. Two traces below each histogram indicate time courses of amplitude modulation $(A M)$ and differential phase modulation $(D P M)$. Vertical thin lines at the left end of the histograms represent 100 spikes/sec for $A_{2-7}$, and $25 \mathrm{spikes/sec} \mathrm{for} B_{2-6}$. Vertical broken lines mark location of $p$, indicating response peaks. Small inset histograms show distribution of response spikes in the carrier signal cycle. Numbers below these histograms indicate degree of phase locking, $v$ (see Materials and Methods). $A$, An E-type neuron; $A_{1}$, photomicrograph; $A_{2-7}$, response histograms from this neuron. $A_{2}, \mathrm{~S}_{1}$ and $\mathrm{S}_{2}$ were applied with a frequency difference $\mathrm{Df}=-2 \mathrm{~Hz}$. $A_{3}, \mathrm{~S}_{1}$ and $\mathrm{S}_{2}$ were applied with a frequency difference $\mathrm{Df}=+2 \mathrm{~Hz}$. Note different temporal combinations of amplitude and phase modulations shown by the two traces below the histograms. $A_{4-7}, \mathrm{~S}_{2}$ was turned off and $\mathrm{S}_{1}$ was modulated only in amplitude. Depth of amplitude modulation was made progressively smaller. $B_{1-6}$, An I-type neuron. Responses in $A_{4-6}$ and $B_{4,5}$ are statistically significant at $p<0.01$ (Rayleigh's test).

sponded solely to amplitude modulation (Fig. 2). This can be seen by comparing two histograms for negative and positive Dfs that have an identical and synchronous time course for amplitude modulation but a $180^{\circ}$-shifted time course for different signs of Df. Differences of locations of histogram peaks, expressed by $p$ (see Materials and Methods), between the histograms for positive and negative Dfs were $<30^{\circ}$ for all neurons. Thus, we conclude that these neurons are sensitive exclusively to amplitude modulation.

These neurons were sensitive to small-amplitude modulation. All neurons showed strong responses to amplitude modulation of $5 \%$. Some neurons showed significant responses to $0.5 \%$ of amplitude modulation (Rayleigh's test; Batschelet, 1965) (Fig. 2).

Five of 17 neurons responded to amplitude increases or ampli- 

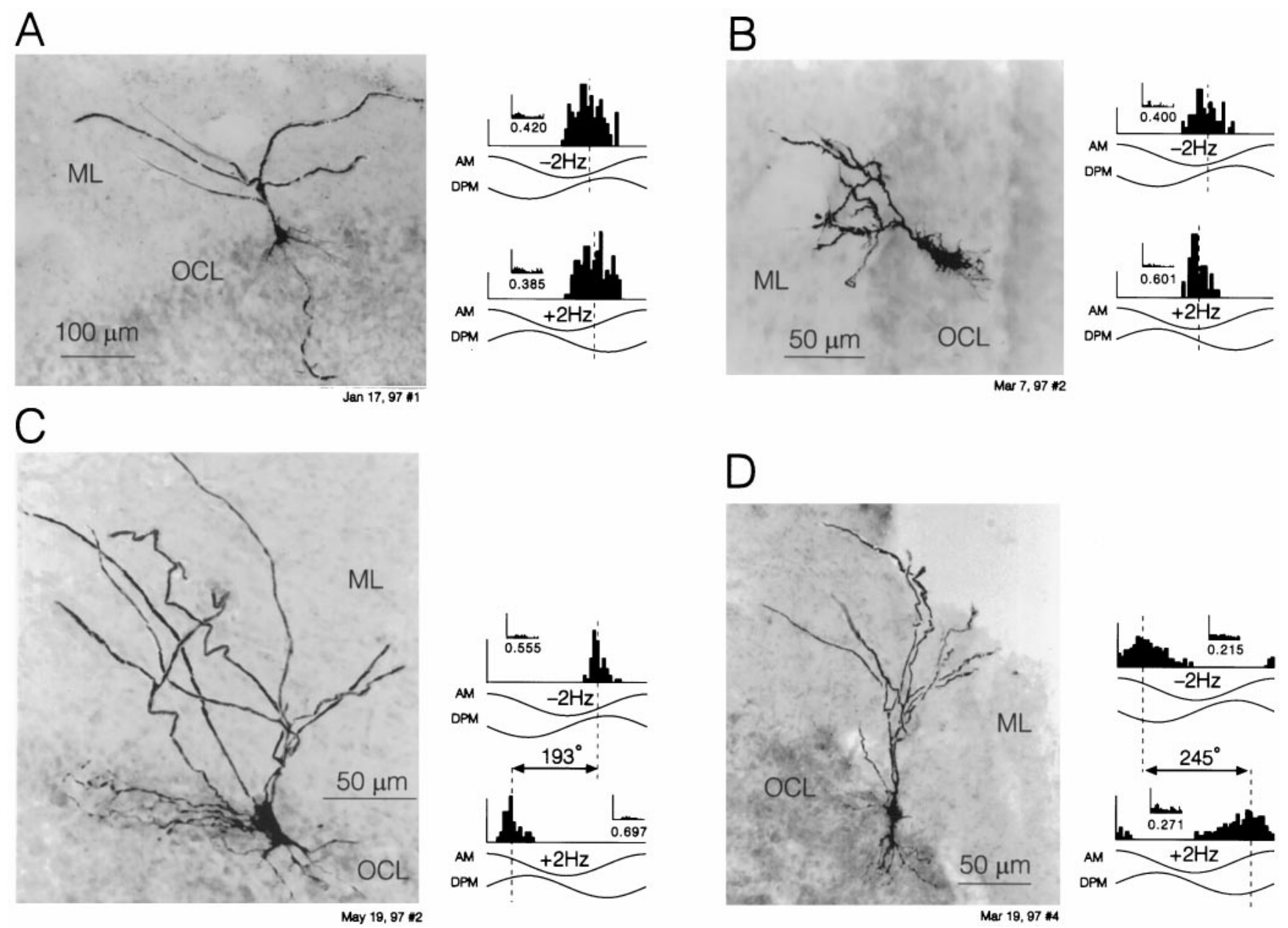

Figure 3. Neurons recorded in OCL of MZ. A, An E-type neuron. Note that response peaks occur during the rising phase of amplitude modulation regardless of differential phase modulation. $B$, An I-type neuron responding at the amplitude troughs regardless of differential phase. $C$, A differential phase-sensitive neuron. Note that response peak shifts by $\sim 180^{\circ}$ when the sign of Df is switched. $D$, A neuron that responded to both amplitude and differential phase modulation. Note that the response peak was shifted by $245^{\circ}$. Vertical thin lines at the left end of histograms indicate 30 spikes/sec in $A$ and $B, 50$ spikes/sec in $C$, and 100 spikes/sec in $D$.

tude peaks (E-type, Fig. $2 A$ ), and the remaining 12 responded to amplitude decreases or responded at amplitude troughs (I-type, Fig. $2 B)$. Both types of neurons had similar somata $(21.5 \pm 4.74$ $\mu \mathrm{m}$ in diameter) and apical dendrites (1.8-2.0- $\mu \mathrm{m}$-thick). The apical dendrite consists of a few proximal dendrites that further ramify into $\sim 12$ distal dendrites that penetrate into ML. The distal dendrites are $\sim 0.5$ - $\mu \mathrm{m}$-thick, and the dendritic field covers several hundred microns. Basal dendrites are larger and penetrate deeper into DFL in E-type neurons $(\sim 250-300 \mu \mathrm{m})$ than in I-type neurons $(\sim 150-200 \mu \mathrm{m})$. All neurons of tuberous type in DZ were projection neurons that send their axons out of ELL via DFL to the midbrain (see below).

\section{Neurons in $M Z$}

Seventy-one neurons were labeled in $\mathrm{MZ}$ using the $\mathrm{S}_{1} / \mathrm{S}_{2}$ stimulus regimen. Of these, 55 were labeled in OCL and 16 in ICL.

\section{OCL of $M Z$}

The neurons found in this area $(n=55)$ were classified into four categories based on their responsiveness to amplitude and differential phase modulation. Twelve neurons were categorized as sensitive only to amplitude modulation, as were the tuberous neurons in DZ. Ten neurons were categorized as sensitive solely to differential phase modulation, and 22 neurons responded to both amplitude and differential phase modulations. The remaining 11 neurons were of the ampullary type.

Figure $3 A, B$ shows an E-type and an I-type neuron that were sensitive solely to amplitude modulation. Much as in neurons in DZ, difference of $p$ in their modulation cycle histograms for negative and positive Df was $<30^{\circ}(n=12)$. These neurons had soma diameters of $23.1 \pm 5.3 \mu \mathrm{m}$. Soma locations of I-type neurons $(n=6)$ tended to be deeper than E-type neurons $(n=6)$. The dendritic field for the basal dendrites of I-type neurons tended to be larger (up to $500 \mu \mathrm{m}$ in diameter) than that of E-type neurons and may spread deeper into OFL (the neuron shown in Fig. $3 B$ is atypical in this regard). Their apical main dendrite $(3-\mu \mathrm{m}$-thick) branches into several and then to $>10$ distal processes covering several hundred microns into ML.

The second type of neurons responded only to differential phase modulation. As presented in Figure $3 C$, the response peak moved by $\sim 180^{\circ}$ when the sign of Df was switched, indicating that the response peaks appeared at a nearly same point in the differential phase function (lower traces of modulation sinusoids in the figure). Neurons were categorized as purely differential phase-sensitive when the difference of $p$ between negative- and 


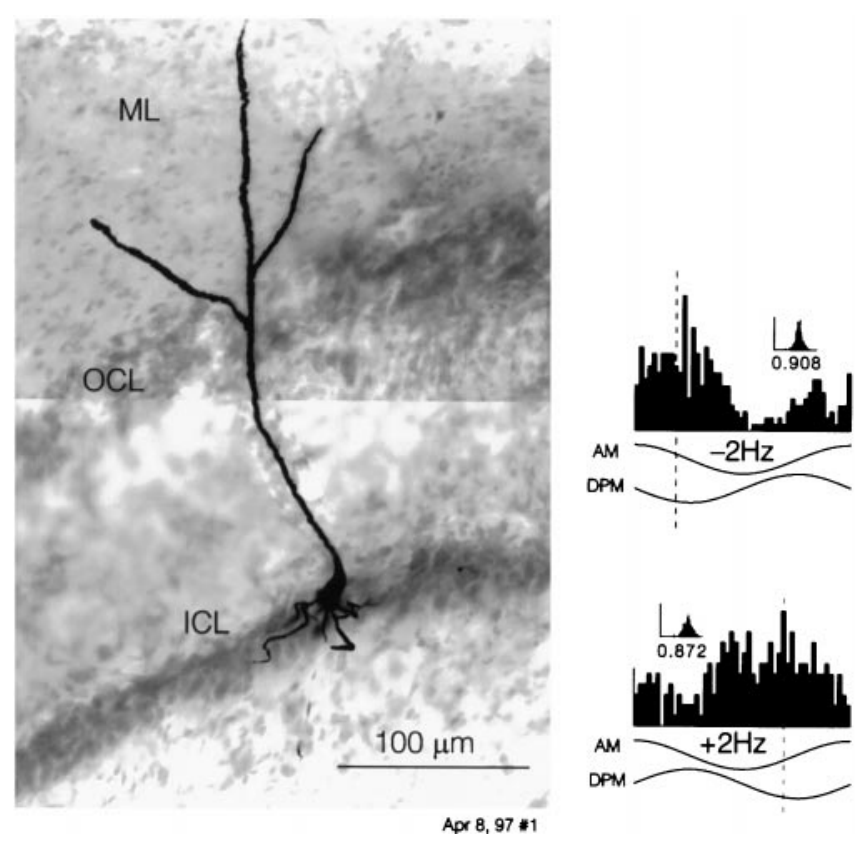

Figure 4. A differential phase-sensitive neuron in ICL of MZ. Note the shift of response peak by $\sim 180^{\circ}$. Also note high values of $v$ indicating a high degree of spike synchronization to the carrier signal. Vertical thin lines: 100 spikes/sec.

positive-Df modulation histograms was $180^{\circ} \pm 30^{\circ}(n=10)$. These neurons also had a large apical dendritic tree in ML. Soma diameter was $19.3 \pm 5.1 \mu \mathrm{m}$. Their basal dendrites spread horizontally over several hundred microns but were confined in OCL and did not penetrate into OFL.

Somata of amplitude-sensitive neurons were more deeply located in the OCL than those of differential phase-sensitive neurons. If the depth of somata is expressed by percentage of distances between outer edge of OCL and inner edge of ICL, soma depths measured from the outer edge of OCL were $20.8 \pm 7.7 \%$ for amplitude-sensitive neurons, and $6.7 \pm 9.3 \%$ for differential phase-sensitive neurons.

The third type of neurons $(n=22)$ responded to both amplitude and differential phase modulations. The neuron shown in Figure $3 D$ shifted its response peak $(p)$ in the modulation cycle histograms by $245^{\circ}$ when the sign of Df was switched. This neuron primarily responded to differential phase modulation (a $180^{\circ} \mathrm{shift}$ is expected) but also preferred larger amplitude, shifting the response peaks by $65^{\circ}$ toward the points at which amplitude was largest. Some other neurons responded to amplitude and differential phase modulations equally well by shifting response peaks by $\sim 90^{\circ}$. This last type of neuron was further investigated physiologically in the phase chamber (see below) in which amplitude and differential phase modulation could be independently manipulated.

The last type of neurons $(n=11)$ responded to low-frequency signals (see below).

\section{ICL of $M Z$}

Sixteen neurons were recorded and labeled in ICL of MZ. Seven of those responded solely to differential phase modulation (Fig. 4 ), with the difference of $p$ between negative-Df- and positiveDf-modulation histograms being $180^{\circ} \pm 30^{\circ}$, as in the phasesensitive neurons in OCL. Eight neurons, however, showed larger shifts of response peaks in modulation histograms for negative and positive Dfs, indicating the influence of amplitude modulation. This neuron type was further studied in the phase chamber (see below). These two physiological types did not appear to differ in their morphology. Their soma diameter was $16.0 \pm 5.6$ $\mu \mathrm{m}$, and they also have apical and basal dendrites. The dendritic morphology differed from that of OCL neurons. The primary apical dendrite was thicker $(\sim 3 \mu \mathrm{m})$ and penetrated straight into OFL, OCL, and ML. Only three to five thick $(\sim 3 \mu \mathrm{m})$ branches emerged from the primary dendrite after it entered ML. The basal dendrites were short but thicker and spread along the boundary between ICL and DFL where giant neurons terminate (Kawasaki and Guo, 1996).

One exceptional neuron in ICL responded exclusively to amplitude modulation. This neuron showed similar morphology to the rest of the ICL neurons.

\section{Experiments using the phase chamber}

As mentioned above, some neurons in $\mathrm{MZ}$ appeared to respond to both amplitude and differential phase modulation when tested with the $\mathrm{S}_{1} / \mathrm{S}_{2}$ signal. Because these neurons are potentially important for the control of JAR, which requires temporal analysis of amplitude and differential phase modulation, they were further investigated using the phase chamber in which the head and trunk areas of the animal were electrically isolated so that effects of amplitude modulation and phase differences between head and trunk areas could be individually studied. Twelve neurons in ICL and 19 neurons in OCL were physiologically recorded and successfully labeled in these experiments.

Figure $5 A$ shows responses of a neuron that was later anatomically identified in ICL. The situation in which the EOD frequency of the animal is $1 \mathrm{~Hz}$ lower than that of a neighbor (Df = $+1 \mathrm{~Hz}$ ) is emulated in Figure $5 A_{1}$ by presenting a particular combination of amplitude and phase modulations in the head compartment, whereas no modulation is presented in the trunk compartment. In contrast, Figure $5 A_{2}$ emulates the situation in which Df $=-1 \mathrm{~Hz}$. If the neuron responded solely to amplitude modulation, the response peak in the histograms in Figure 5, $A_{1}$ and $A_{2}$, should have occurred at the same position. Conversely, if the neuron responded solely to differential phase modulation, the peak should have moved by $180^{\circ}$, as indicated by the stimulus traces for phase (broken lines). In reality, the peak moved by $\sim 120^{\circ}$ in this neuron, indicating that both amplitude and phase modulation affected the response. Similar results were obtained when the signals of the head and trunk compartments were swapped (Figs. $5 A_{3}, A_{4}$ ).

Experiments in Figure 5, $A_{5}$ and $A_{6}$, show that this neuron is sensitive to phase differences between the two chambers because it responded equally to a phase advance of the head and to a phase delay of the trunk. Responses in Figure 5, $A_{5}$ and $A_{6}$, disappeared when the signal of the unmodulated compartment was turned off or both compartments were stimulated with the identical phase modulations (data not shown). In Figure $5, A_{7}$ and $A_{8}$, phase modulation was turned off, and effects of amplitude modulation alone were examined. When amplitude modulation was given in the head compartment, the neuron responded at amplitude trough (Fig. 5 $A_{7}$ ), whereas the neuron responded to amplitude peak when it was applied to the trunk compartment (Fig. $5 A_{8}$ ). This amplitude-induced response completely disappeared when the unmodulated signal in the opposite compartment was turned off (Fig. 5 $A_{9}$ ). Other neurons shown in Figure 5, $B$ and $C$, are similar, except that the effect of amplitude modulation was smaller in the head compartment in the neuron pre- 


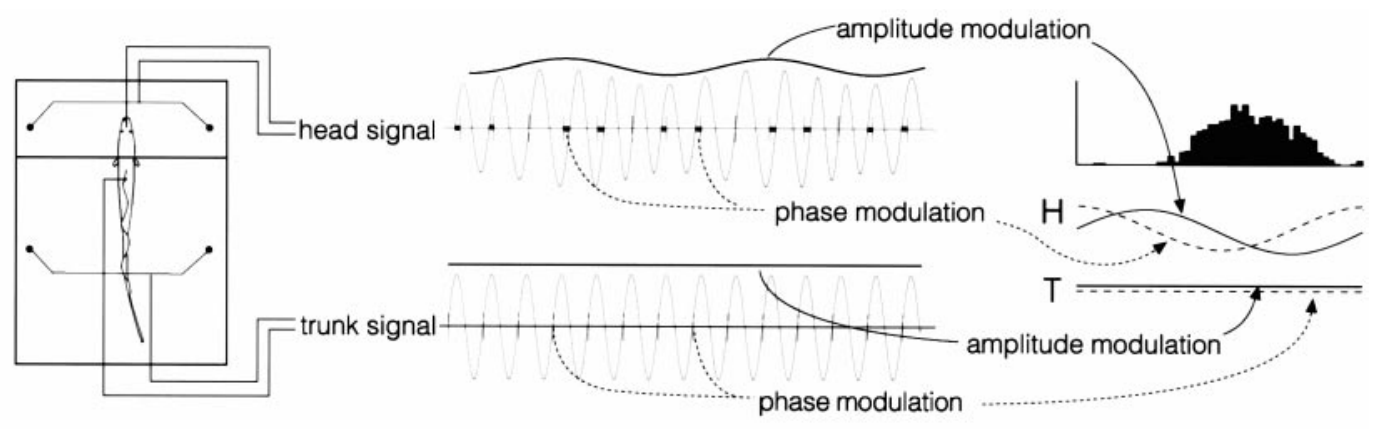

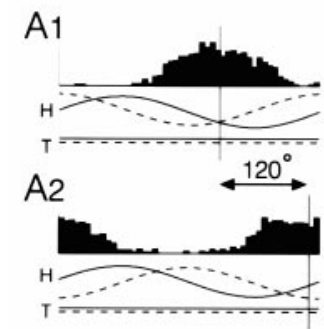

A3

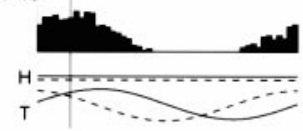

A4

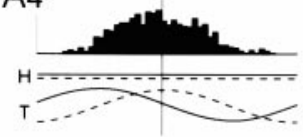

A5
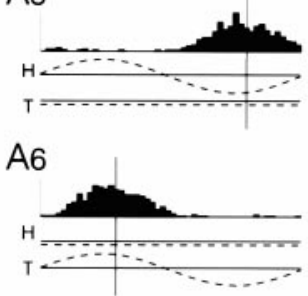

A7

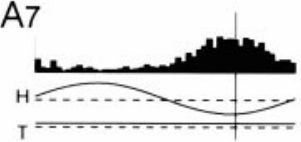

A8

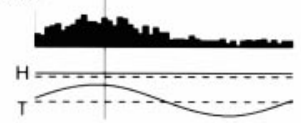

A9
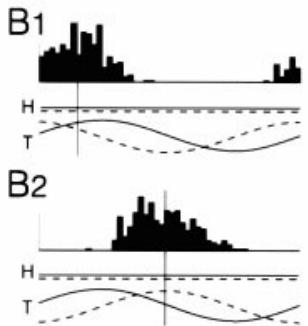

B3

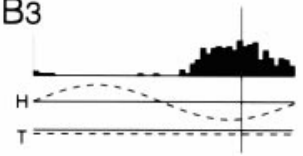

B4

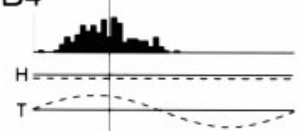

B5

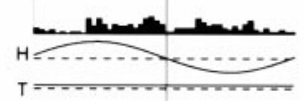

B6

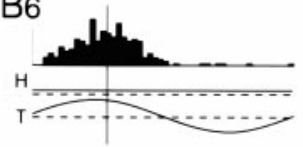

B7

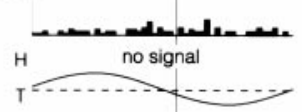

C1

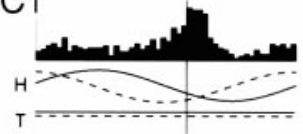

C2

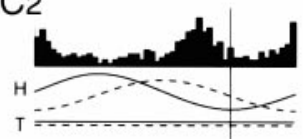

C3
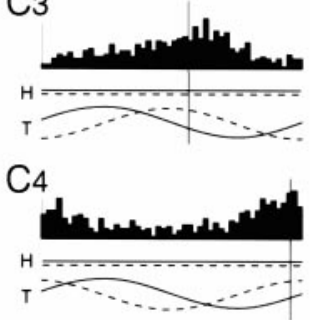

C5
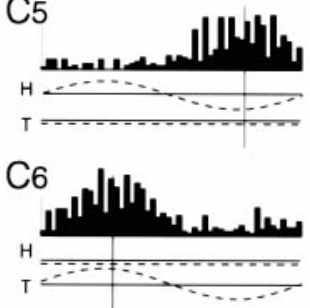

C7

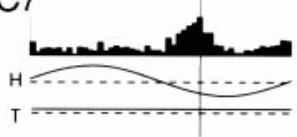

C8

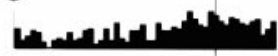

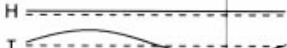

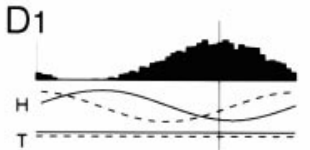

D2

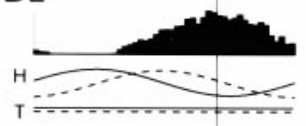

D3
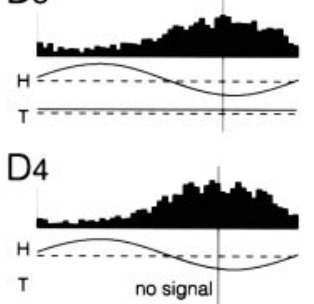

D5

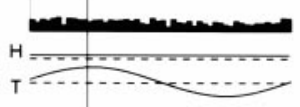

D6

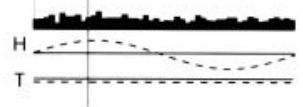

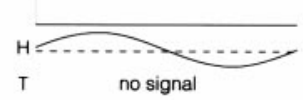

Figure 5. Four example neurons tested with the phase chamber. Each column of histograms represents data from one neuron. Top left, Head and trunk of fish were isolated electrically by a partition $(>40 \mathrm{~dB})$. Top center, Each compartment was given a sinusoidal stimulus whose amplitude and phase were independently controlled. Top right, Explanation of stimulus traces under the histograms presented below: top two traces represent signals in the head compartment $(H)$, bottom two traces represent signals in the trunk $(T)$. Solid and broken lines, respectively, indicate amplitude and phase modulation. Phase advance is plotted upward. $A-C$, Differential phase-sensitive neurons in MZ that respond to phase difference between the two compartments (compare $\left.A_{5,6}, B_{3,4}, C_{5,6}\right)$. Amplitude modulation also induces responses $\left(A_{7,8}, B_{6}\right.$, and $\left.C_{7}\right)$. See Results for detailed explanation. $D$, An amplitudesensitive neuron in DZ that responded only to amplitude modulation in the head compartment $\left(D_{1-4}\right)$. Amplitude modulation in the trunk $\left(D_{5}\right)$ or phase modulation anywhere $\left(D_{1}, D_{2}, D_{6}\right)$ had no effect. Vertical thin lines: 50 spikes/sec for all histograms. 
Table 1. Vector strength in different neuron types

\begin{tabular}{llllc} 
Area & Layer & Physiology & Vector strength, $v$ & $\begin{array}{c}\text { Number of } \\
\text { neurons }\end{array}$ \\
\hline DZ & OCL & AM-sensitive & $0.169 \pm 0.143$ & 8 \\
MZ & ICL & DPM-sensitive & $0.914 \pm 0.050$ & 20 \\
MZ & OCL & AM-sensitive & $0.210 \pm 0.092$ & 20 \\
MZ & OCL & DPM-sensitive & $0.503 \pm 0.145$ & 20
\end{tabular}

Vector strength, $v$, was computed in each neuron. The stimulus that evoked the best response was used to compute $v$. AM, Amplitude modulation, DPM, differential phase modulation.

sented in Figure $5 B$ and in the trunk compartment in Figure $5 C$. Phase-locked neurons that provide input to the differential phasesensitive neurons are not completely indifferent to amplitude modulation because their firing latency is often slightly shorter for stimulus of large amplitude (see Discussion). All other ICL neurons recorded with the phase chamber were differential phasesensitive and showed responses to pure amplitude modulation, although the degree of responses to amplitude modulation varied significantly. In some neurons the sensitive area was limited to one of the compartments. Morphology and location of these ICL neurons labeled in the phase-chamber experiments were indistinguishable from those of neurons labeled in experiments with the $\mathrm{S}_{1} / \mathrm{S}_{2}$ stimulus regimen.

Neurons found in OCL in the phase-chamber experiments were categorized into two groups, differential phase-sensitive $(n=12)$ and amplitude-sensitive $(n=7)$, as in the $\mathrm{S}_{1} / \mathrm{S}_{2}$ experiments. Response properties of the differential phase-sensitive neurons in OCL were similar to those in ICL. Figure $5 B$ shows an example that showed responses to amplitude alone and only in the trunk compartment (Fig. 5, compare $B_{5}, B_{6}$ ). Figure $5 C$ shows another example in which amplitude modulation had an effect primarily in the head compartment. Again, responses to pure amplitude disappeared when the signal in the other compartment was turned off (Fig. $5 B_{7}$ ). Figure $5 D$ shows an OCL neuron that responded solely to amplitude modulation in the head compartment.

Because the interaction of amplitude and differential phase sensitivity may give rise to selectivity for the sign of Df, all neurons recorded in the phase chamber were carefully examined for possible selectivity for the sign of Df. Histograms (repetition $=30$ ) were constructed several times for each sign of Df and the spike frequency of the neuron was computed. Although sensitivity of neurons may change somewhat over several minutes in an unpredictable manner, no systematic and significant difference was observed between two signs of Df in any neuron examined $(n=31)$.

\section{Degrees of phase locking in different types of neurons}

The degree of synchronization of spikes to the carrier signal was computed whenever we constructed histograms for modulation cycles. Each histogram in Figures $2-4$ has an inset histogram that shows distribution of spikes in the cycle of the carrier signal. The vector strength, $v$, is shown below these histograms. The differential phase-sensitive neurons in ICL consistently showed a high degree of synchronization. The differential phase-sensitive neurons in OCL showed an intermediate degree of synchronization. Amplitude-sensitive neurons in DZ and MZ showed the lowest degree of synchronization (Table 1).

\section{Projection of tuberous type ELL neurons}

Intense labeling was shown in $\sim 80 \%$ of tuberous-type neurons into which biocytin was attempted to be injected. Without excep- tion, these intensely labeled neurons possessed an axon that exited the ELL. In the rest of the tuberous-type neurons, only a part of the dendritic tree was weakly labeled. We did not find labeled axons or labeled somata in these cases. We found a projecting axon in all tuberous-type neurons that showed good labeling of somata and dendrites.

All neurons in DZ and the ICL of MZ were found to send their axons into DFL. Some neurons in OCL of MZ similarly send axons into DFL, but axons of other neurons run along OFL ventrally before exiting ELL. Projection of ELL neurons is shown in Figure 6. All of these axons entered the ELL commissure, which connects two halves of the ELL and contains commissural axons of giant cells (Kawasaki and Guo, 1996). The axons left the ELL commissure ventrolaterally immediately after crossing the midline. This contralateral projection ran toward the lateral end of the brain and then turned anteriorly toward the motor nucleus of the trigeminal (Fig. $6 \mathrm{~A}, \mathrm{mV}$ ). In the majority of cases, the axon gave off an ipsilateral branch of a few hundred microns before crossing the midline (Fig. 6C). In some cases, however, no ipsilateral branch was seen despite strong labeling of the axon. This type of exclusively contralateral projection was confirmed in a differential phase-sensitive neuron in OCL of MZ. Other cases in which no ipsilateral branching was observed were not conclusive because faint staining of the axons was often the case at this level.

The ipsilateral axons ran along a more medial path than the contralateral ones. At the level of the PE, the contralateral path from ELL neurons in one side merged with ipsilateral paths from neurons in the opposite sides of the ELL. There, most of the neurons (all of ICL and most of amplitude and phase neurons in OCL) gave off a collateral that projected in the PE. The collateral spreads a large $(600-700 \mu \mathrm{m})$ terminal field within PE (Fig. 6D). Projection to the PE was confirmed from both contralateral and ipsilateral axons. The main axons of 23 neurons were intensely labeled and could be followed further to the end of their projection terminals in the torus. In one I-type neuron in DZ, labeling of the axon was intense at the level of PE, but neither contralateral nor ipsilateral branch gave projection to PE. No neuron was found to exclusively project to PE.

After passing the region of $\mathrm{PE}$, the axon bundle ran into the ventral border of the torus. Thereafter, the paths were neurontype dependent. Axons of all DZ neurons entered the LP subdivision of the torus. No axon terminals of DZ neurons were found to project to other parts of the torus. Within LP all observed axons spread extensively as they reached the lateral end of the nucleus. In contrast, the projection of axons of all MZ neurons was exclusively to the MD and LA subdivisions of the torus. The axon recursively branched into numerous fine terminal processes (0.3-0.5- $\mu \mathrm{m}$-thick) that reached the surface of the torus. The terminal spread was generally extensive (up to $1 \mathrm{~mm}$ )(Fig. $6 E$ ). 

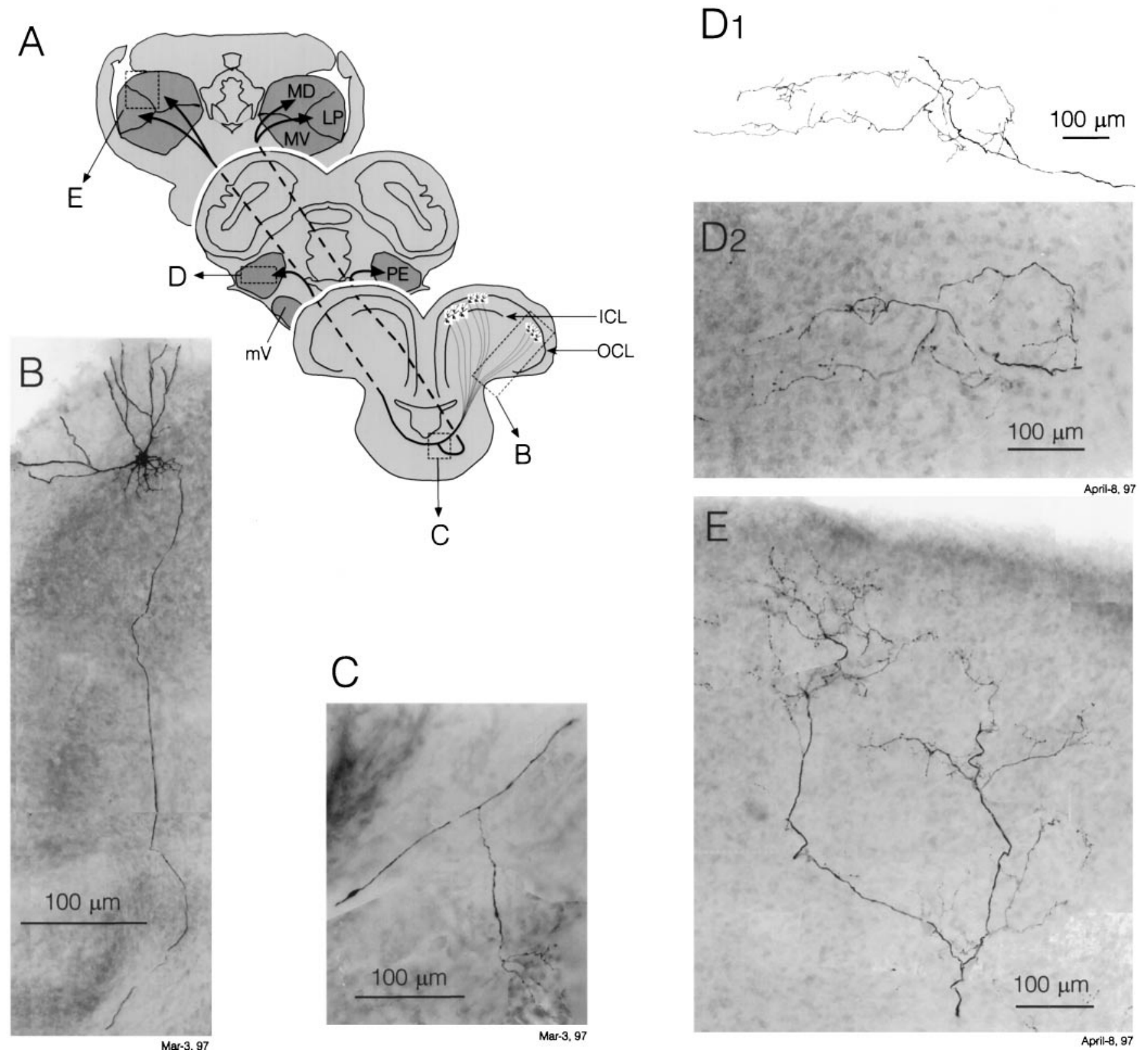

Figure 6. Projection paths and their terminals of ELL neurons revealed by whole-cell recording and labeling with biocytin. $A$, A majority of neurons send their axons to both contralateral and ipsilateral pathways (broken lines) and terminate in both the PE and the torus. Broken-line rectangles indicate approximate location of example photomicrographs shown in $B-E$. $B$, An amplitude-sensitive neuron in DZ is sending its axon in DF. $C$, This axon gives off an ipsilateral branch before it reaches the midline. The projection terminals seen in the right lower corner in $C$ were observed only in this neuron. $D, E$, Projection terminals of the differential phase-sensitive neuron in ICL that is shown in Figure $4 . D_{l}$, Camera lucida drawing of terminal branches in the PE. $D_{2}$, A montage photomicrograph from two 100- $\mu \mathrm{m}$ thick sections containing the same terminal branches. $E$, Contralateral terminal branches and their endings in the torus. A montage photomicrograph from different focal planes in five consecutive 100- $\mu \mathrm{m}$-thick sections.

\section{Ampullary neurons}

During the course of study of tuberous-type ELL neurons, we encountered neurons that could not be driven either by amplitude or differential phase modulation, or by a combination of them. Some of these neurons strongly responded when an EOD mimic was replaced with a $1 \mathrm{~Hz}$ sinusoidal signal. Although the ampullary electrosensory system was not the primary focus of this study, occurrence of these neurons is reported here because of their novel location (see Discussion).

Two of these neurons were found in OCL of MZ and had apical dendrites that penetrated into ML much as tuberous-type neu- rons. One of them responded to the voltage-decreasing slope of the signal, and the other neuron responded to the opposite slope (Fig. 7). Only the former neuron was labeled strongly enough to follow its axon, which terminated in the MD of the torus. The projection was observed only in the contralateral torus, but not in the PE.

Other neurons $(n=4)$ had soma $(\sim 13 \mu \mathrm{m})$ in DZ or in MZ (Fig. $7 B$ ). They had many branches of proximal dendrites that were highly ramified (field size, $\sim 1000 \mu \mathrm{m}$ ). Their axons bifurcated at $\sim 200 \mu \mathrm{m}$ from the soma. The ipsilateral axon branch extensively ramified and spread in OFL of MZ. The other branch 

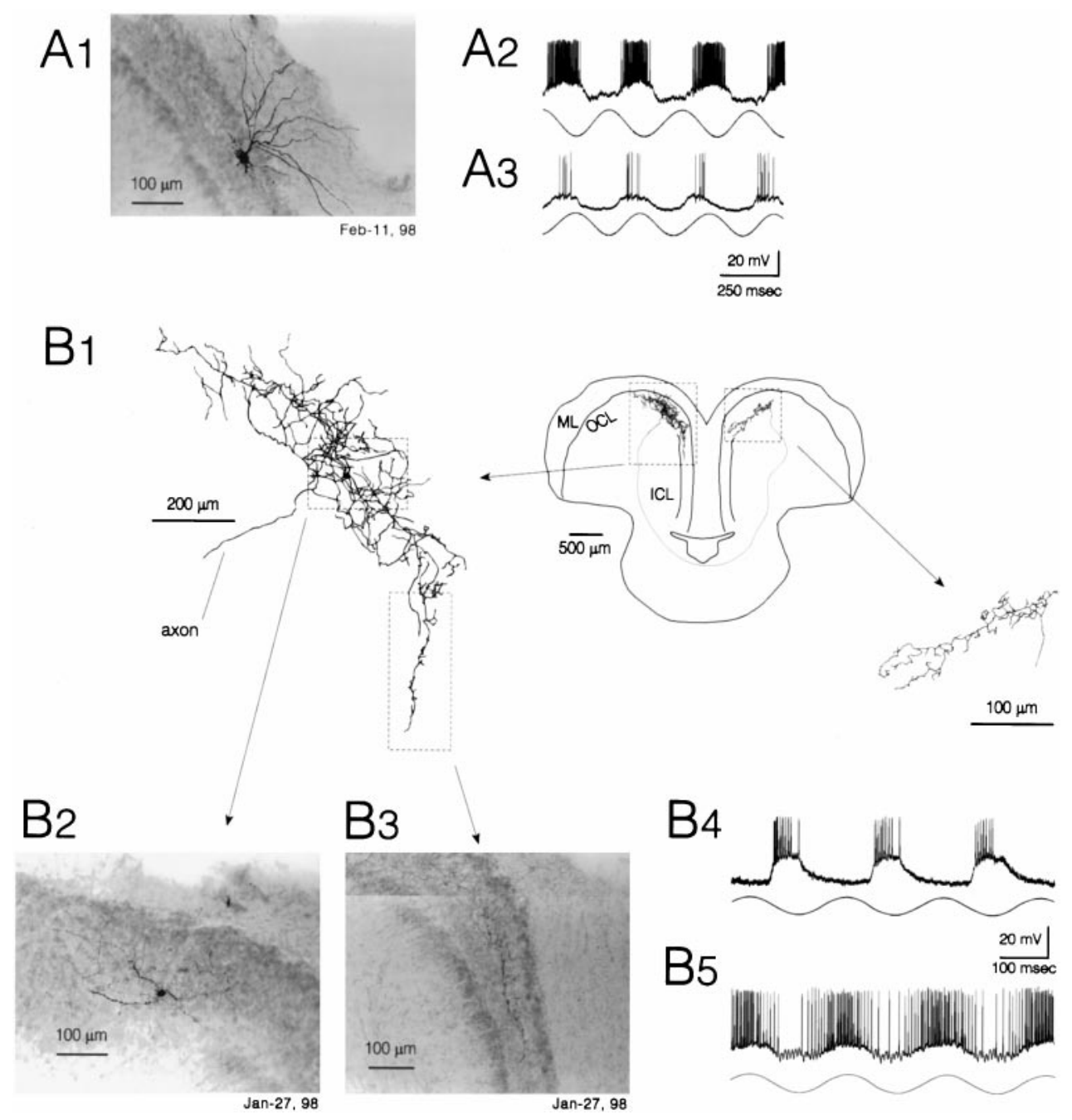

Figure 7. Low-frequency-sensitive neurons found in the ELL. $A_{1}$, Neuron with apical dendrites in ML. $A_{2}$, Voltage trace of whole-cell recording from this neuron showed responses to decreasing slopes of stimulus voltage. $A_{3}$, Voltage trace of another neuron showing preference to voltage increasing slope. The neuron for the trace in $A_{3}$ also had apical dendrites in ML (morphology not shown because of lack of complete labeling). Sinusoidal traces at the bottom in $A_{2}$ and $A_{3}$ indicate stimulus voltage $(2 \mathrm{~Hz}, 0.2 \mathrm{mV} / \mathrm{cm}) . B$, A commissural neuron in ELL. $B_{1}$, Camera lucida drawing of the neuron that was sensitive to voltage-decreasing slope. Processes surrounding the soma (in the left ELL) extended $1000 \mu \mathrm{m}$ in the depth of the drawing. The contralateral terminal field (in the right ELL) consisted of thin $(0.3 \mu \mathrm{m})$ processes and extended $600 \mu \mathrm{m}$ in the depth of the drawing. Photomicrographs of two parts of the neuron are shown in $B_{2}$ and $B_{3} . B_{4}$, Voltage trace of responses of this neuron to $5 \mathrm{~Hz}$ signal $(0.5 \mathrm{mV} / \mathrm{cm})$ showing preference to voltage-decreasing slopes. $B_{5}$, Response of another neuron showing preference to voltage-increasing slopes.

ran into OFL, crossed the midline, and reached the corresponding contralateral area. There the axon ramified into a large $(\sim 600$ $\mu \mathrm{m})$ terminal field. These neurons showed preferences either for negative or positive slope of signal voltage and did not send axons to the midbrain.

Ten other neurons that strongly responded to a low-frequency sinusoidal signal were found in DZ and MZ, but the labeling of these neurons was not complete and only their soma (diameter, $11.67 \pm 2.89 \mu \mathrm{m})$ and a part of their processes were seen. Projection areas could not be identified in these partially filled neurons.

The ampullary-type neurons occurred in the same areas in DZ and $\mathrm{MZ}$ in which we labeled tuberous-type neurons.

We did not penetrate the $\mathrm{VZ}$ and, thus, the type of neurons that exist in the VZ of Gymnarchus is unknown.

\section{DISCUSSION}

Major findings of this study are as follows: (1) distribution of neuron types within the ELL has been determined (Fig. 8); (2) information on amplitude and differential phase is represented separately by different neurons in the ELL; (3) nonlinear interactions between amplitude and differential phase information, which are required for the jamming avoidance response, do not occur within the ELL; (4) DZ of the ELL is dedicated to processing amplitude information; (5) OCL of MZ contains both amplitude-sensitive neurons and differential phase-sensitive neurons; and (6) these ELL neurons project to the torus semicircularis and the nucleus praeeminentialis of the midbrain in a partially overlapping manner.

A behavioral study (Kawasaki, 1993) demonstrated that Gymnarchus, when it encounters a jamming neighbor, determines whether it should raise or lower its own discharge frequency by analyzing temporal patterns of amplitude and differential phase modulation. As shown in Figures 2 and 3, some neurons in ELL are purely sensitive to either amplitude or differential phase modulation, representing separate processing of these two cues. Amplitude and phase information are respectively sampled by 


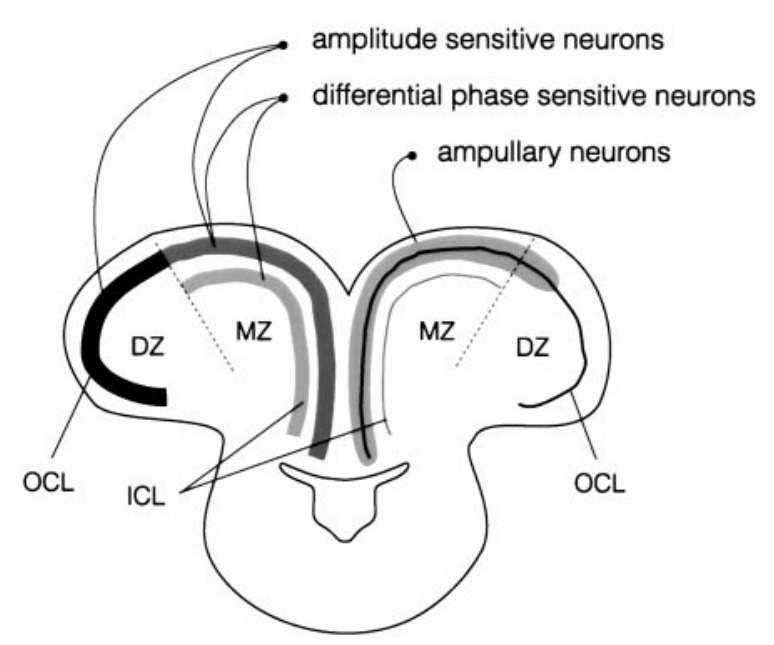

Figure 8. Summary diagram showing areas of the ELL and neuron types. Only amplitude-sensitive neurons were found in the OCL of DZ. Only differential phase-sensitive neurons were found in the ICL of MZ. Both types of neuron were found in the OCL of MZ. Ampullary type neurons were found in the OCL of both $\mathrm{MZ}$ and DZ.

O-type and S-type afferent fibers (Bullock et al., 1975). Although S-type fibers project to the ICL of MZ (Kawasaki and Guo, 1996), the projection pattern of O-type fibers within ELL has not been determined yet. Labeling of individual O-type fibers is required to determine whether the same fibers project to both areas.

The present finding that the amplitude-sensitive neurons in DZ and OCL of MZ project in different areas in the torus suggests that they may have different functions. Different behavioral roles and physiological properties of areas in ELL have been shown in a gymnotiform electric fish, Eigenmannia (Shumway, 1989a,b; Metzner and Juranek, 1997). As in other groups of fishes [Mormyrids (Bell and Grant, 1992); Gymnotiformes (Scheich, 1977; Bastian, 1981); and catfish (McCreery, 1977)], Gymnarchus have both E-type and I-type amplitude-sensitive neurons in ELL. Both types of neurons, particularly in DZ, are highly sensitive to small-amplitude modulation $(\sim 0.5 \%)$, as shown in Figure 2 . Behavioral experiments using the jamming avoidance response demonstrated that Gymnarchus can detect $0.02 \%$ of amplitude modulation (Guo and Kawasaki, 1997).

Differential phase-sensitive neurons found by our previous study using sharp intracellular electrodes (Kawasaki and Guo, 1996) were confirmed in the current study by a whole-cell patch recording and labeling technique. This technique also allowed us to label axons to their terminals in the midbrain. The differential phase-sensitive neurons in both ICL and OCL projected solely to the midbrain, and we did not observe any axon collaterals that projected within the ELL. Based on single-cell labeling of input elements, Kawasaki and Guo (1996) suggested that differential phase-sensitive neurons in ICL of MZ directly receive phaselocked inputs from S-type afferents and giant neurons, but phaselocked neurons do not reach OCL of MZ. Thus, the differential phase-sensitive neurons in OCL must receive differential phase information via another interneuron or from the differential phase-sensitive neurons in ICL without axonal conduction. The putative interneurons could not be recorded in this study, perhaps because of the sampling bias of our whole-cell patch electrodes. Sequential processing of differential phase information by neurons in ICL and OCL is supported by distinct differences in the degree of synchronization of spikes to stimulus cycles (Table 1).
These differential phase-sensitive neurons often showed responsiveness also to amplitude modulation. The amplitude sensitivity is explained by the amplitude-dependent phase shift of phase-locked afferent fibers (Kawasaki and Guo, 1996, their Fig. $12 G)$. These phase-locked afferent fibers show slightly shorter latency for increased amplitude of the stimulus, and this latency shift in turn stimulates the differential phase neurons. Figure $5, A_{5}$ and $A_{6}$, show that this neuron is sensitive to delayed phase in the head or advanced phase in the trunk. Responses to amplitude modulation in Figure $5, A_{7}$ and $A_{8}$, can be interpreted as responses to phase shifts induced by amplitude modulation (compare $A_{5}, A_{7}$, and $A_{6}, A_{8}$ ). The notion that the responses in Figure 5, $A_{7}$ and $A_{8}$, are responses to an amplitude-induced phase difference is further supported by the fact that the neuron ceases to respond to amplitude modulation when the signal in the other compartment is turned off (compare $A_{7}, A_{9}$ ). In some neurons, the effect of amplitude modulation in one compartment was small or nonexistent (Fig. $5 B_{5}, C_{7}$ ). In these neurons, the amplitudelatency function for one of the compartments must be flat, as shown by some of the phase-locked fibers (Kawasaki and Guo, 1996, their Fig. 12G), which explains why the shift of the histogram peak in Figure 5, $C_{1}$ and $C_{2}$, is smaller than that in Figure $5, C_{3}$ and $C_{4}$. From these observations, we conclude that the neurons in ELL that are sensitive to both amplitude and differential phase receive only phase information, and the amplitude information carried by O-type afferent fibers does not reach these neurons. A similar intensity-phase trade-off is known in binaural phase-sensitive neurons in the inferior colliculus of the cat $(\mathrm{Ku}$ wada and Yin, 1983; Yin and Kuwada, 1983).

Control of the jamming avoidance response requires neurons that integrate amplitude and differential phase information. The integration should be nonlinear in nature because different temporal patterns of identical inputs must yield different responses (Kawasaki, 1993). The integration of responses to amplitude and differential phase modulations in the ambiguous neurons discussed here, however, is linear: the responses to separately presented amplitude and differential phase stimulus largely predict the responses to the simultaneous presentation of component signals. In fact, none of the neurons recorded in ELL in this study show selectivity to different patterns of amplitude and phase modulation that represent different signs of Df. The LP and LA of the torus, a common projection area of amplitude and differential phase-sensitive neurons of the ELL, is a likely area in which neurons that are selective to the sign of Df might be found.

All tuberous neurons recorded in this study were of similar morphological type with large apical dendrites, clearly reflecting the sampling bias of the whole-cell recording technique used in this study. Although the size of postsynaptic potentials sometimes reached $30 \mathrm{mV}$, they were usually relatively small $(10-15 \mathrm{mV})$, whereas resting potentials were always large $(-40$ to $-50 \mathrm{mV})$, indicating that our recording was often made in the apical dendrites.

This study also demonstrated that the projection pattern of ELL neurons to the midbrain in Gymnarchus is vastly different from that of closely related pulse-type mormyrid fishes. In Gymnarchus, phase comparison is performed in the ELL, and amplitude and differential phase-sensitive neurons coexist in the same region (MZ) of the ELL. These amplitude and differential phasesensitive neurons commonly project to LA and MD of the torus. DZ of the ELL is dedicated to amplitude processing. In contrast, in pulse-type mormyrid fishes, the phase comparison is performed in the nucleus exterolateralis of the torus semicircularis 
that receives phase-locked input via a distinct region of the ELL, the nucleus of electrosensory lateral line lobe (Amagai, 1998; Amagai et al., 1998; Friedman and Hopkins, 1998). In the ELL of mormyrid, the amplitude coding A-type fibers terminate in the MZ, whereas phase-sensitive B-type fibers terminate in DZ (Maler et al., 1973a,b; Bell et al., 1981, 1989; Bell, 1990a,b; von der Emde and Bell, 1994). These comparisons indicate that organization of sensory processing can be rather different even between closely related species (Kawasaki, 1996).

Differential phase-sensitive neurons in the ELL were previously shown to be sensitive to small phase differences on the order of microseconds (Guo and Kawasaki, 1997; Kawasaki, 1997). Likewise, amplitude-sensitive neurons in the ELL were found to be sensitive to small-amplitude depths of modulation in this study. Both amplitude and phase difference change their center values of modulation in natural conditions. The large apical dendrites of ELL neurons, which are demonstrated to be involved in descending control in other electric fishes (Bell et al., 1997), may be also involved in a mechanism that sets a working range of the neurons to the center value of the modulation. The projection of ELL neurons in Gymnarchus to the PE is likely to be involved in the descending pathway (Bastian and Bratton, 1990).

Neurons sensitive to low-frequency signals $(<50 \mathrm{~Hz})$ have been found intermingled with tuberous-type neurons in MZ and DZ in this study of Gymnarchus. This is a unique form of neural organization among electric fish species. Neurons sensitive to lowfrequency signals were found exclusively in the VZ in closely related mormyrid fishes (Bell and Russell, 1978; Bell, 1981) and in remotely related gymnotiform electric fishes; low-frequencysensitive neurons are also found in a dedicated area, the medial zone of the ELL (Maler et al., 1974; Heiligenberg and Dye, 1982; Shumway, 1989a,b).

An independently evolved South American electric fish, Eigenmannia, performs similar JAR (Heiligenberg, 1991). Despite the independent evolution, the computational algorithms for JAR in Gymnarchus are remarkably similar to those in Eigenmannia (Kawasaki, 1993). Nevertheless, different forms of neuronal implementation of the same computational tasks are being discovered (Kawasaki, 1996). For example, different brain structures, the hindbrain in Gymnarchus and the midbrain in Eigenmannia, compute differential phase information (Carr et al., 1986; Kawasaki and Guo, 1996). The current study showed that the two tasks for JAR, separate processing and integration of amplitude and phase information, are performed by different brain structures in Gymnarchus, whereas these tasks are accomplished by a layered nucleus in the midbrain in Eigenmannia (Rose and Heiligenberg, 1985).

\section{REFERENCES}

Amagai S (1998) Time coding in the midbrain of mormyrid electric fish. II. Stimulus selectivity in the nucleus exterolateralis pars posterior. J Comp Physiol 182:131-143.

Amagai S, Friedman MA, Hopkins CD (1998) Time coding in the midbrain of mormyrid electric fish. I. Physiology and anatomy of cells in the nucleus exterolateralis pars anterior. J Comp Physiol 182:115-130.

Bass AH, Hopkins CD (1982) Comparative aspects of brain organization of an African "wave" electric fish, Gymnarchus niloticus. J Morphol 174:313-334.

Bastian J (1981) Electrolocation. II. The effects of moving objects and other electrical stimuli on the activities of two categories of posterior lateral line lobe cells in Apteronotus albifrons. J Comp Physiol 144:481-494.

Bastian J, Bratton B (1990) Descending control of electroreception. I. Properties of nucleus praeeminentialis neurons projecting indirectly to the electrosensory lateral line lobe. J Neurosci 10:1226-1240.
Batschelet E (1965) Statistical methods for the analysis of problems in animal orientation and certain biological rhythms. Washington, DC: American Institute of Biological Sciences.

Bell C, Bodznick D, Montgomery J, Bastian J (1997) The generation and subtraction of sensory expectations within cerebellum-like structures. Brain Behav Evol 50:17-31.

Bell CC (1981) Central distribution of octavolateral afferents and efferents in a teleost (mormyridae). J Comp Neurol 195:391-414.

Bell CC (1990a) Mormyromast electroreceptor organs and their afferent fibers in mormyrid fish. II. Intra-axonal recordings show initial stages of central processing. J Neurophysiol 63:303-318.

Bell CC (1990b) Mormyromast electroreceptor organs and their afferent fibers in mormyrid fish. III. Physiological differences between two morphological types of fibers. J Neurophysiol 63:319-332.

Bell CC, Grant K (1992) Sensory processing and corollary discharge effects in mormyromast regions of mormyrid electrosensory lobe. II. Cell types and corollary discharge plasticity. J Neurophysiol 68:859-875.

Bell CC, Russell CJ (1978) Termination of electroreceptor and mechanical lateral line afferents in the mormyrid acousticolateral area. J Comp Neurol 182:367-382.

Bell CC, Finger TE, Russell CJ (1981) Central connections of the posterior lateral line lobe in mormyrid fish. Exp Brain Res 42:9-22.

Bell CC, Zakon H, Finger TE (1989) Mormyromast electroreceptor organs and their afferent fibers in mormyrid fish: I. Morphology. J Comp Neurol 286:391-407.

Bullock TH, Behrend K, Heiligenberg W (1975) Comparison of the jamming avoidance responses in Gymnotoid and Gymnarchid electric fish: a case of convergent evolution of behavior and its sensory basis. J Comp Physiol 103:97-121.

Carr CE, Heiligenberg W, Rose GJ (1986) A time-comparison circuit in the electric fish midbrain. I. Behavior and physiology. J Neurosci 6:107-119.

Friedman MA, Hopkins CD (1998) Neural substrates for species recognition in the time-coding electrosensory pathway of mormyrid electric fish. J Neurosci 18:1171-1185.

Goldberg JM, Brown PB (1969) Response of binaural neurons of dog superior olivary complex to dichotic tonal stimuli: some physiological mechanisms of sound localization. J Neurophysiol 32:613-636.

Guo Y-X, Kawasaki M (1997) Representation of accurate temporal information in the electrosensory system of the African electric fish, Gymnarchus niloticus. J Neurosci 17:1761-1768.

Heiligenberg W (1975) Electrolocation and jamming avoidance in the electric fish Gymnarchus niloticus (Gymnarchidae, Mormyriformes). J Comp Physiol 103:55-67.

Heiligenberg W (1991) Neural nets in electric fish. Cambridge, MA: MIT.

Heiligenberg W, Dye J (1982) Labeling of electroreceptive afferents in gymnotid fish by intracellular injection of HRP: the mystery of multiple maps. J Comp Physiol 148:287-296.

Heiligenberg W, Rose G (1985) Phase and amplitude computations in the midbrain of an electric fish: intracellular studies of neurons participating in the jamming avoidance response of Eigenmannia. J Neurosci 5:515-531.

Hopkins CD (1974) Electric communication in fish. Am Sci 62:426-437.

Kawasaki M (1993) Independently evolved jamming avoidance responses employ identical computational algorithms: a behavioral study of the African electric fish, Gymnarchus niloticus. J Comp Physiol 173:9-22.

Kawasaki M (1994) The African wave-type electric fish, Gymnarchus niloticus, lacks corollary discharge mechanisms for electrosensory gating. J Comp Physiol 174:133-144.

Kawasaki M (1996) Comparative analysis of the jamming avoidance response in African and South American wave-type fishes. Biol Bull 191:103-108.

Kawasaki M (1997) Sensory hyperacuity in the jamming avoidance response of weakly electric fish. Curr Opin Neurobiol 7:473-479.

Kawasaki M, Guo Y-X (1996) Neuronal circuitry for comparison of timing in the electrosensory lateral line lobe of an African wave-type electric fish, Gymnarchus niloticus. J Neurosci 16:380-391.

Kuwada S, Yin T (1983) Binaural interaction in low-frequency neurons in inferior colliculus of the cat. I. Effects of interaural delays, intensity and repetition rate on interaural delay function. J Neurophysiol 50:981-999. 
Lissmann HW (1958) On the function and evolution of electric organs in fish. J Exp Biol 35:156-191.

Lissmann HW, Machin KE (1958) The mechanism of object location in Gymnarchus niloticus and similar fish. J Exp Biol 35:451-486.

Maler L, Finger T, Karten H (1974) Differential projection of ordinary lateral line receptors and electroreceptors in the gymnotiform fish, Apteronotus albifrons. J Comp Neurol 158:363-382.

Maler L, Karten H, Bennett M (1973a) The central connections of the anterior lateral line nerve of Gnathonemus petersii. J Comp Neurol 151:67-84.

Maler L, Karten H, Bennett M (1973b) The central connections of the posterior lateral line nerve of Gnathonemus petersii. J Comp Neurol 151:57-66.

McCreery D (1977) Two types of electroreceptive lateral lenmiscal neurons of the lateral line lobe of the catfish, Ictalurus nebulosus; connections from the lateral line nerve and steady-state frequency response characteristics. J Comp Physiol 113:317-339.

Metzner W, Juranek J (1997) A sensory brain map for each behavior? Proc Natl Acad Sci USA 94:14798-14803.

Rose G, Fortune E (1996) New techniques for making whole-cell recordings from CNS neurons in vivo. Neurosci Res 26:89-94.
Rose GJ, Heiligenberg W (1985) Structure and function of electrosensory neurons in the torus semicircularis of Eigenmannia: morphological correlates of phase and amplitude sensitivity. J Neurosci $5: 2269-2280$

Scheich H (1977) Neural basis of communication in the high frequency electric fish, Eigenmannia virescens (jamming avoidance response). III. Central integration in the sensory pathway and control of the pacemaker. J Comp Physiol 113:229-255.

Shumway CA (1989a) Multiple electrosensory maps in the medulla of weakly electric gymnotiform fish. I. Physiological differences. J Neurosci 9:4388-4399.

Shumway CA (1989b) Multiple electrosensory maps in the medulla of weakly electric gymnotiform fish. II. Anatomical differences. J Neurosci 9:4400-4415.

von der Emde G, Bell CC (1994) Responses of cells in the mormyrid electrosensory lobe to EODs with distorted wave-forms: implications for capacitance detection. J Comp Physiol 175:83-93.

Yin T, Kuwada S (1983) Binaural interaction in low-frequency neurons in inferior colliculus of the cat. II. Effects of changing rate and direction of interaural phase. J Neurophysiol 50:1000-1019. 